\title{
Cyber physical systems in mechatronic research centre
}

\author{
Timotei István Erdei ${ }^{1}$, Zsolt Molnár ${ }^{2}$, Nwachukwu C. Obinna ${ }^{3}$, Géza Husi ${ }^{4}$ \\ ${ }^{1}$ University of Debrecen, timoteierdei@gmail.com, Hungary \\ ${ }^{2}$ University of Debrecen, zsolt.molnar94@gmail.com, Hungary \\ ${ }^{3}$ University of Debrecen, nwachukwucobinna@gmail.com, Hungary \\ ${ }^{4}$ University of Debrecen, husigeza@eng.unideb.hu, Hungary
}

\begin{abstract}
In the Building Mechatronic Research Centre we started to develop our cyberphysical system. The Department provided us all necessary equipment to realize the first cyberphysical system. The main core of the project was to create an augmented reality based navigation system in our robot laboratory. In that aspect we also built an internet of things ready automated guided vehicle prototype. It must be said that the internet of things has induced a new paradigm shift in the socio-economic world. Nowadays, augmented reality and virtual reality are industrial processes development tools. In recent years, these technologies demonstrated significant improvements in real-time industrial technology.
\end{abstract}

\section{Introduction}

As result the efficiency of maintenance tasks may depreciate. In order to increase the efficiency in this area, augmented reality and virtual reality technology may be used.

The maintenance includes a range of complex industrial tasks and categorized into administrative, technical and management processes.

Augmented reality technology contains digital data and other technical details and is able to provide information about industrial machinery-equipment, without disassembling the equipment.

It is possible to read various data within the equipment, during its operation and in real-time. This facilitates analysis of "black box" systems.

With augmented reality technology we can measure and display the measurement data on a head-up display.

Augmented Reality enables, abnormalities to be detected quickly without special installation skills and propose several repair strategies.

\section{Open computer vision based image processing}

The department's robotic laboratory includes a KUKA
KR5 medium payload industrial welding robot, KUKA KR3, FANUC spider and Sony scara SRX-611 and we wanted to create a unique system.

Our main goal was realizing a cyber-physical system for an industrial robot laboratory and find alternative ways of internet of things.

The head of our department G. Husi [1] had written a detailed documentation about industry 4.0. The document include the new technologies even augmented reality, virtual reality and other new industrial processes, that guided us to create our cyber-physical system.

The data and parameters have been encoded into quick response codes and other unique markers.

In the first test we use these codes as position markers and an instruction set to control a prototype automated guided vehicle robot.

The automated guided vehicle used an internet protocol based camera as vision sensors.

The Building Mechatronic Research Centre features several functional internet protocol cameras, which were interfaced to our cyber-physical system.

Behind the internet protocol cameras we used a relatively strong desktop computer. The computer has an Intel Corei7 central processing unit, 16 gigabyte random access memory, 2 terabyte hard disk drive \& 2 x Nvidia GeForce GTX 650 Titan. On the other hand we chose the open computer vision which is vision library software with numerous algorithms for image processing, M. Marengoni [2].

\footnotetext{
Corresponding author: timoteierdei@gmail.com
} 
In order to implement the augmented reality algorithm, a special plugin/library known as augmented reality marker targeted was required.

Augmented reality gives an informative view of the world, and the main parts include a camera, J. Rubinstein [3], quick response codes with hidden information and image processing software to decode it.

With a quick response code we can detect position in real-time.

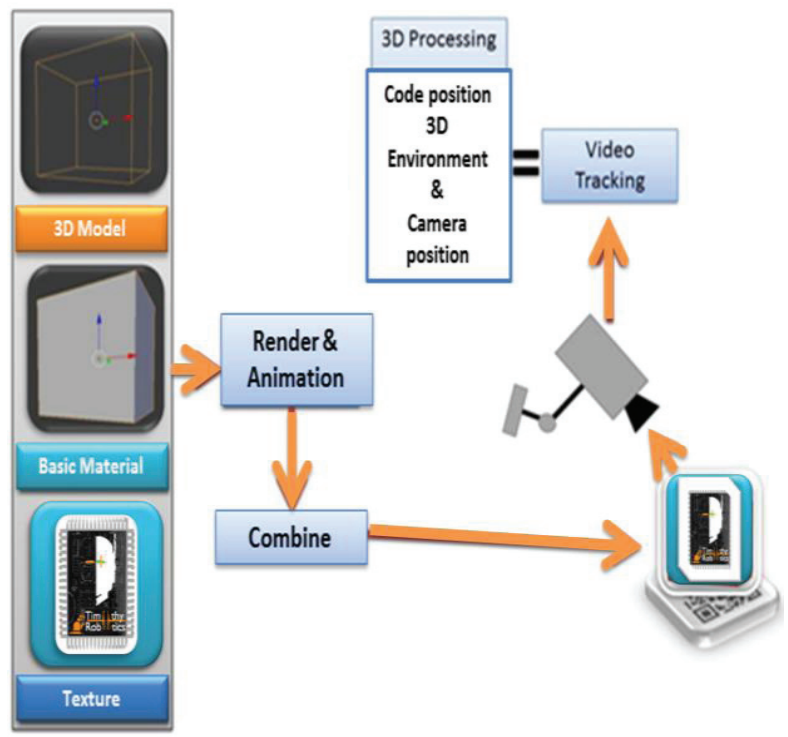

Fig. 1. Augmented reality and open computer vision

As Fig. 1. represents this type of image processing is a complex task. The proposed augmented reality system requires understanding the motion of a point in a 3 dimension coordinate system as can be seen on Fig. 2 .

The field of view of a camera is a world that is seen, and detected with sensors, A. L. Kaczmarek [4].

The detection process involves the measurement of an object's velocity.

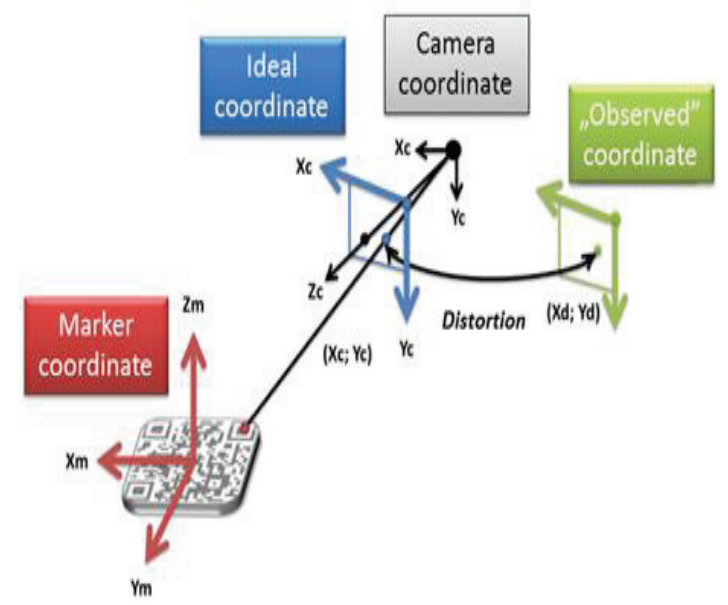

Fig. 2. Camera view and position detection of object

This is a complex method because first you need to design the 3 dimension model in a 3 dimension computer aided design software, and after it you need to decrease the polygon number of the 3 dimension model.

High polygon number values are computationally expensive. First we design all 3 dimension models in a

3 dimension computer aided design software, and after it with we decreased the numbers of the polygons to make our program run smoother, R. Inkulu [5].
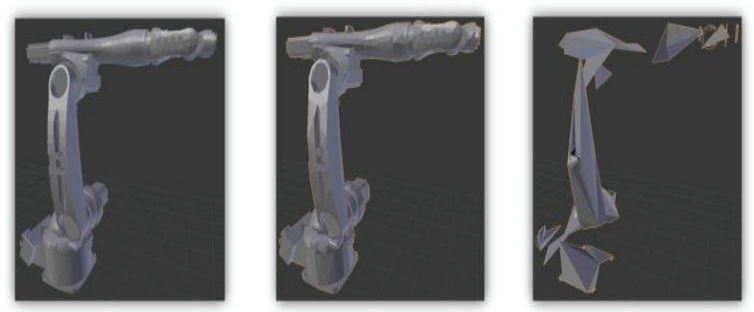

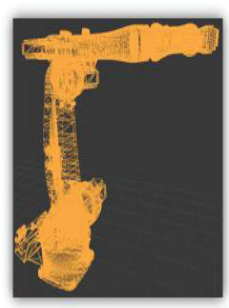

50000 Polygon

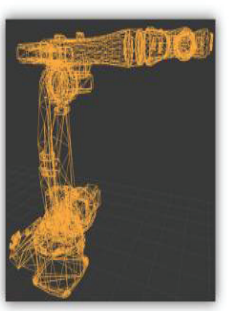

25000 Polygon

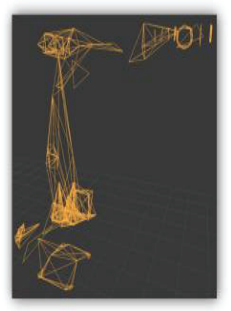

1000 Polygon
Fig. 3. Polygon reduction

We can confirm that polygon reduction on the Fig. 3. can be effective. The next step of our research was designing the automated guided vehicle prototype.

The automated guided vehicle system would include trajectory planning and tracking, as well as collision avoidance. The most important point of the robot is there are no sensors on the robot itself, it uses only the internet protocol cameras of the robotics laboratory for trajectory tracking.

Firstly we designed the automated guided vehicle concept in a 3 dimension computer aided design modeling software and made a part lists with the necessary equipment, G. Balaji [6].

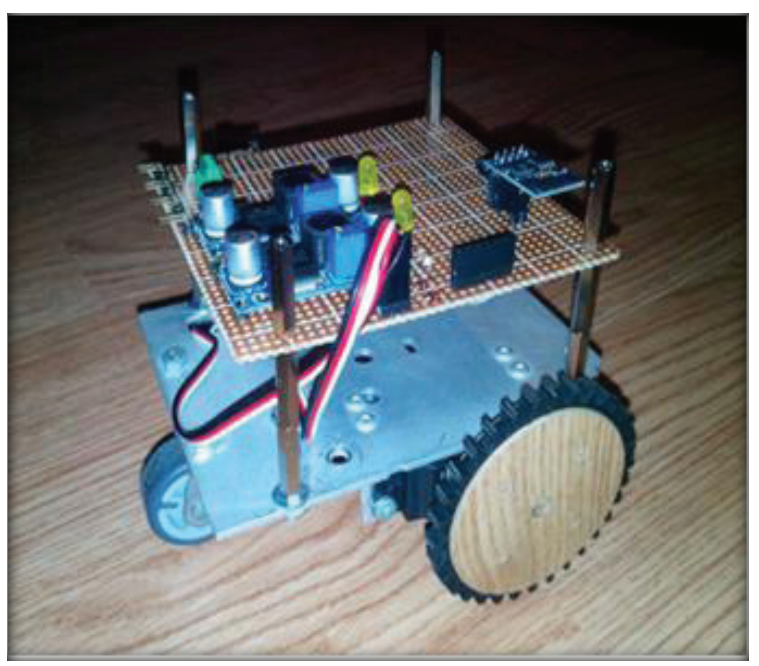

Fig. 4. Augmented reality and open computer vision 
Automated guided vehicle prototype robot on Fig. 4. has an Arduino Nano, M. Banzi [7], two LM2596 direct current step down converter and a wireless fidelity to serial module.

\section{Augmented reality navigation system}

In the first part of our project we found a suitable place to test the system which was the robotics laboratory in the Building Mechatronic Research Centre. We designed the quick response codes/markers and printed it out in different sizes, and placed them in the right order to make the path for the robot.

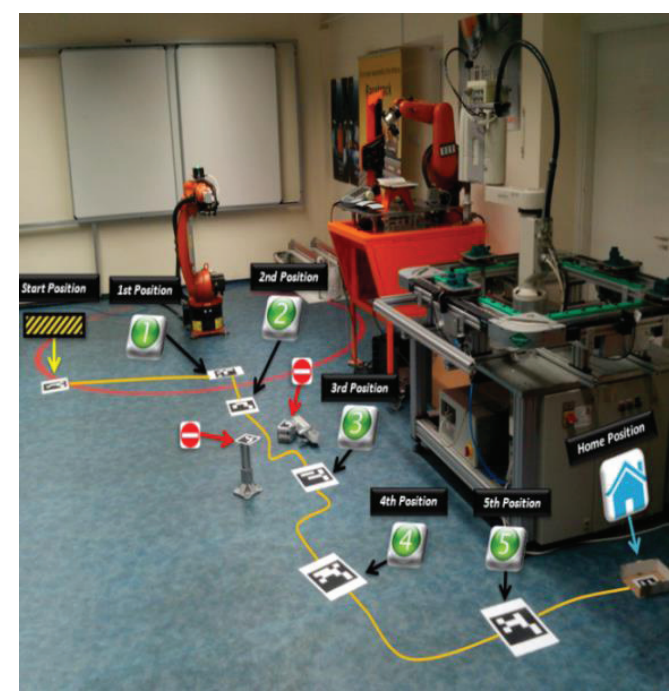

Fig. 5. Robot laboratory as isolated space

The task of the automated guided vehicle prototype robot is to leave the "Start" position go to the 1st position and wait until the KUKA KR5 goes to the position of the automated guided vehicle and put on it the steel cube. After it reach the 2nd, 3rd 4rd, 5rd positon and the Home position with the cargo. We printed a code for identifying the automated guided vehicle's orientation.

The route used for testing is represented by Fig. 5. above. The internet protocol cameras of robot laboratory see the codes and streams data to the notebook via wireless fidelity. On the desktop computer, open computer vision and augmented reality marker targeted software was required recognizes the position of the code and decodes it, sends the information to the automated guided vehicle prototype robot which plans and tracks the trajectory.

In the first test we launched/programmed the KUKA KR5 industrial robot manipulator with our electronic printed circuit board, name TiMo board, to control the robot's gripper.

Furthermore we established a new wireless fidelity network, name TP-Link router to make the wireless fidelity signal stronger in the Robot Laboratory it was very important because we sent all signals trough wireless fidelity and we wanted to avoid the lag of the stream of the mobile phone.

For the first time we noticed lag in the Desktop computer, fortunately we could set the dedicated Nvidia graphic card to calculate instead of the Corei7 central processing unit which was slower in this instance. The Fig. 6. shows the communication topology used in the robot laboratory.

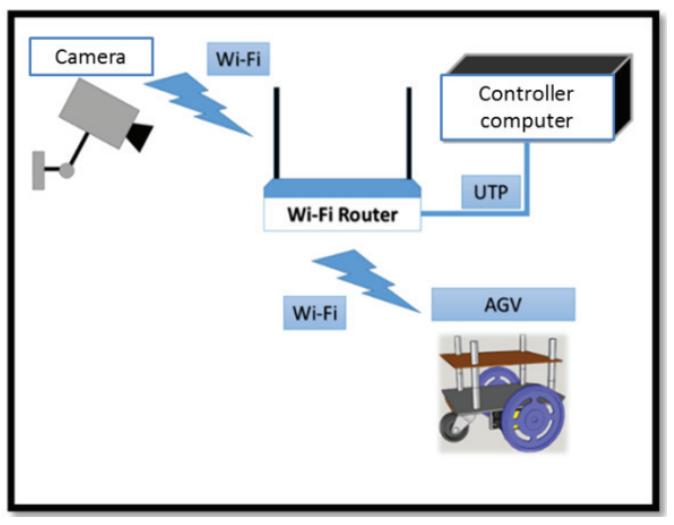

Fig. 6. Network communication system

When we began the first test, the automated guided vehicle prototype robot was in the "Start" position.

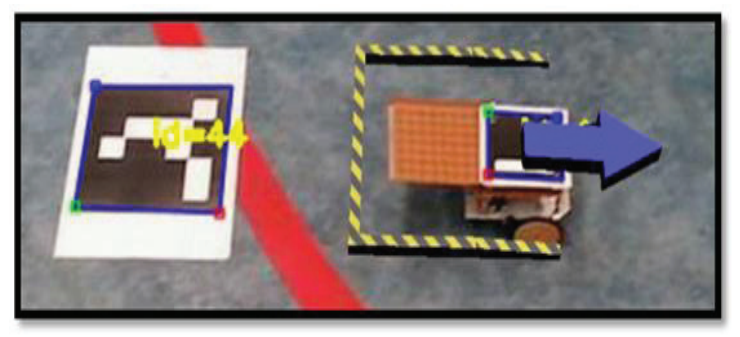

Fig. 7. Start position

After it the automated guided vehicle started from the "Start" position as we can see in Fig. 7., is heading towards to the 1 st position and waited there until KUKA KR5 moved to the automated guided vehicle position and opened the gripper to put the steel cube on the automated guided vehicle.

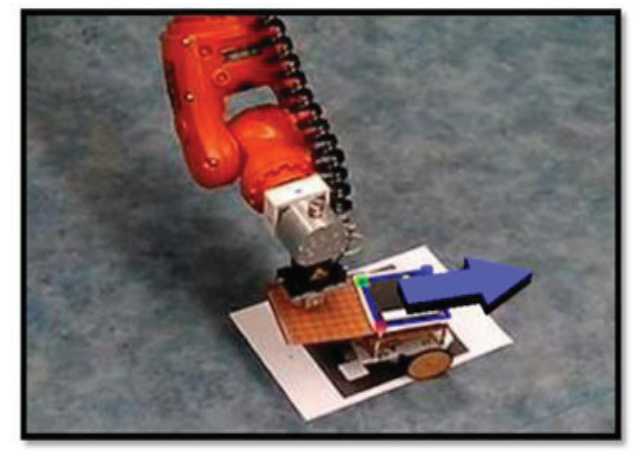

Fig. 8. 1st position

\footnotetext{
${ }^{*}$ Corresponding author: timoteierdei@gmail.com
} 
In the Fig. 8. we can see the robot reaching the 1 st position. The next step was to reach the 3rd positon marker and avoid the collision with the other objects.

To realize this we made codes with other 3D models and we set the Augmented Reality marker targeted a distance away from the other objects, and the automated guided vehicle could avoid them during the motion. When the automated guided vehicle reached the HOME positon it stopped.

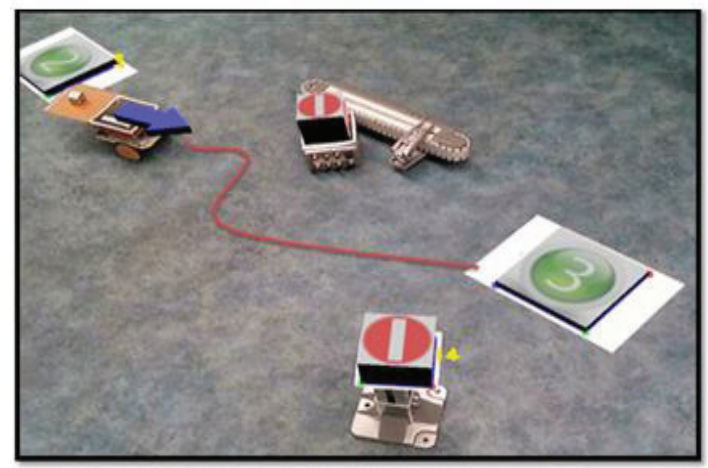

Fig. 9. Automated guided vehicle prototype first test

On Fig. 9. represents that the system can be flexible as navigation system. Because we can add any type of quick response code markers and hide commands various type.

Furthermore the robot did not have any sensors. The only sensors in the building was internet protocol based camera system.

\section{Augmented reality research and development}

In our next project we used quick response code as Augmented Reality marker in order to visualize the position of axis of KUKA KR5 or set position in the workspace of the robot dynamically.

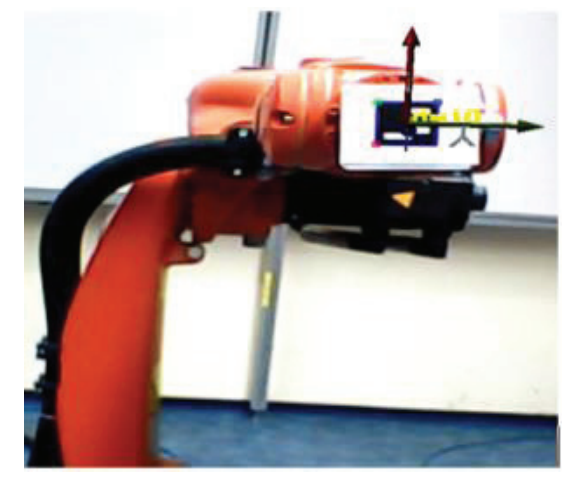

Fig. 10. Augmented reality code to set position visualization

The Augmented Reality technology is also able to test standalone systems and items. We can visualize the tool coordinate system of the robotic arm as we can see in the Fig. 10. above.We developed a gripper to KUKA KR5, we reduced the polygon number of it and mounted "virtually" on to the robot arm.
Furthermore the codes can be established interactive connection between each other.

In one of our tests we used another marker which consist of a column 3 dimension model.

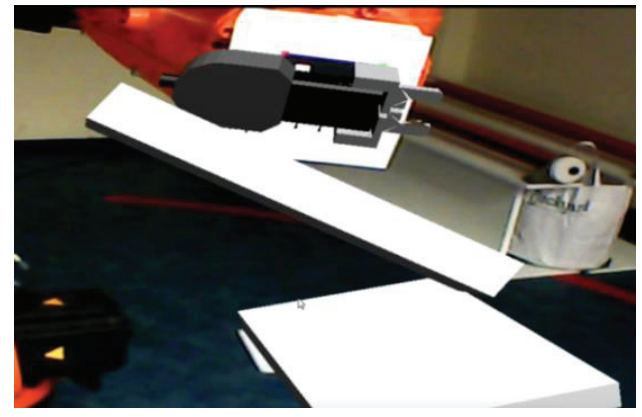

Fig. 11. Virtual augmented reality items and collide

When we moved the real KUKA KR3 with the virtual mounted gripper and it could push away the virtual column in Fig. 11. above.

\section{Conclusion}

During test phase the Augmented Reality based navigation system was stable. We could hide information behind the quick response codes/markers. These information can be commands to any kind of robots in the future. Moreover with this technique we can replace the 3 dimension printer, because we do not need to print out the 3 dimension model itself, we should only hide the 3 dimension model behind quick response codes/markers.

In the future we will create real Automated Guided Vehicle to our Augmented Reality based navigation system.

The project was supported by University of Debrecen, Electrical Engineering and Mechatronics Department.

\section{References}

[1] G. Husi, "Industry 4.0 technology in University of Debrecen", https://www.researchgate.net/publication/301607839_Industry_4 0 Hungarian, accessed on May 2017.

[2] M. Marengoni, "High Level Computer Vision Using OpenCV, Graphics", (Unpublished work style)," unpublished.

[3] J. Rubinstein, "Signal Delay in RC Tree Networks", IEEE Transactions on Computer-Aided Design of Integrated Circuits and Systems, Volume: 2, Issue: 3, July 1983, pages. $202-211$.

[4] A. L. Kaczmarek, "Stereo vision with Equal Baseline Multiple Camera Set (EBMCS) for obtaining depth maps of plants", CEA Volume 135 Issue C, April 2017 pages. 23-37.

[5] R. Inkulu, "Incremental Algorithms to Update Visibility Polygons", Algorithms and Discrete Applied Mathematics, LNCS, Volume: 10156 pages. 205-218.

[6] G. Balaji, "Automated Guided Vehicle Navigated using Fuzzy Cognitive Maps and Intelligent Space" Ijraset Volume 5 Issue III, March 2017 pages 41-44

[7] M. Banzi, "Arduino Nano system", https:/www.arduino.cc/en/uploads/Main/ArduinoNanoManual23 .pdf, accessed on April 2017.

\footnotetext{
${ }^{*}$ Corresponding author: timoteierdei@gmail.com
} 